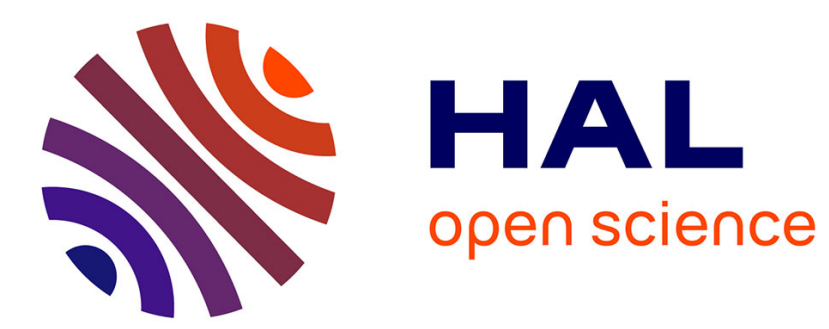

\title{
L'inégale professionnalisation de la vente du vin et de la viande à Toulouse à la fin du Moyen Âge
}

Clémentine Stunault

\section{To cite this version:}

Clémentine Stunault. L'inégale professionnalisation de la vente du vin et de la viande à Toulouse à la fin du Moyen Âge. Rives Méditerranéennes, 2017, 55 (1), pp.79 - 94. 10.4000/rives.5294 . hal01900756

\section{HAL Id: hal-01900756 \\ https://hal.science/hal-01900756}

Submitted on 30 Oct 2018

HAL is a multi-disciplinary open access archive for the deposit and dissemination of scientific research documents, whether they are published or not. The documents may come from teaching and research institutions in France or abroad, or from public or private research centers.
L'archive ouverte pluridisciplinaire HAL, est destinée au dépôt et à la diffusion de documents scientifiques de niveau recherche, publiés ou non, émanant des établissements d'enseignement et de recherche français ou étrangers, des laboratoires publics ou privés. 


\section{L'inégale professionnalisation de la vente du vin et de la viande à Toulouse à la fin du Moyen Âge : quelques éléments d'interprétation}

Le vin et la viande font partie des produits de base de l'alimentation médiévale; leur consommation augmente sensiblement à la fin de la période. Leur vente à Toulouse nous est connue par des sources réglementaires et par quelques rares sources judiciaires émanant de diverses autorités.

$\mathrm{Au} \mathrm{XII}^{\mathrm{e}}$ siècle, la ville est gouvernée par un comte, assisté par des conseillers, les capitouls. Ces derniers dirigent la ville après son rattachement à la couronne de France en 1271. Ils sont toutefois placés sous la surveillance des représentants de l'autorité royale, le sénéchal et son viguier, et leur pouvoir tend à décroître après l'installation du Parlement en 1444.

De nombreux documents nous renseignent sur le commerce de la viande. Il est marqué par une professionnalisation assez précoce, qui se traduit par l'institution de corps de métiers spécialisés et réglementés par la municipalité, et par l'apparition d'experts chargés de contrôler l'ensemble des viandes commercialisées à travers la ville.

La boucherie médiévale a donné lieu à de nombreuses études au cours du $\mathrm{XX}^{\mathrm{e}}$ siècle, les premières portant sur l'organisation de la corporation des bouchers ${ }^{1}$, avant de s'intéresser aux questions d'approvisionnement et de consommation dans les années 1950 à $1970^{2}$. Depuis les années 1990, les historiens se penchent sur le rôle politique des bouchers et sur les nouvelles préoccupations en matière d'hygiène à la fin du Moyen $\hat{A}_{\mathrm{ge}}{ }^{3}$. La viande faisait alors l'objet d'une surveillance attentive, comme l'ont souligné les recherches de Ramón Agustin Banegas López sur Barcelone et celles de Benoît Descamps sur Paris ${ }^{4}$.

$\mathrm{Au}$ contraire, la vente du vin reste méconnue car elle demeure relativement libre : si des professionnels de cette activité se rencontrent dans les sources, ils côtoient de multiples vendeurs non-spécialistes, comme à Bordeaux ou Orvieto ${ }^{5}$.

Les travaux sur le vin se développent à partir des années 1970, portant à la fois sur la culture de la vigne, la production et la commercialisation du vin ${ }^{6}$. Depuis les années 1990 , les publications se multiplient, introduisant de nouveaux questionnements autour des pratiques culturelles liées à la consommation du vin ${ }^{7}$. Cependant, les métiers du vin sont plus difficiles à étudier que ceux de la viande, car ils ont laissé beaucoup moins de sources.

\section{Le marché de la viande : une professionnalisation précoce}

Dès le XII ${ }^{\mathrm{e}}$ siècle, une réglementation est mise en place pour encadrer les activités des bouchers. La préparation et la vente de la viande sont peu à peu réservées à un groupe de professionnels, dotés de compétences spécifiques et reconnus comme tels par la municipalité.

\footnotetext{
${ }^{1}$ Drot, 1902. Duval, 1954. Durbec, 1957.

${ }^{2}$ Dubled, 1967. Favreau, 1968.

${ }^{3}$ Chevalier, 1993. Ferrières, 2002. Giraudet, 2010.

${ }^{4}$ Faugeron, 2006. Banegas López, 2007 et 2012. Descamps, 2009.

${ }^{5}$ Riccetti, 1994. Lavaud, 2003.

${ }^{6}$ Société des historiens médiévistes de l'enseignement supérieur public, 1971. Dion, 1977. Higounet, 1992.

${ }^{7}$ Garrier, 1990 et 1998. Centre culturel de l'abbaye de Flaran, 1991. Gaulin et Grieco, 1994. Lachiver, 1997. Verdon, 2002. Alexandre-Bidon et alii, 2014.
} 


\section{Les premiers signes d'une organisation ( $\mathrm{XII}^{\mathrm{e}}-\mathrm{XIII}^{\mathrm{e}}$ siècles)}

$\mathrm{Au} \mathrm{XII}^{\mathrm{e}}$ siècle, n'importe qui peut découper et vendre de la viande à Toulouse, dans les limites de la sauveté créée entre 1120 et 1140 par le comte Alphonse Jourdain ${ }^{8}$. C'est ce que rappelle en 1195 une déclaration du comte Raymond VI de Toulouse confirmant les privilèges accordés par son aïeul aux habitants de ce territoire ${ }^{9}$. La profession de boucher y est donc libre d'accès.

Le terme «profession » désigne ici une activité rémunérée, exercée régulièrement dans le but de gagner sa vie. Il diffère du terme «métier» qui s'applique à une communauté d'artisans spécialistes d'une activité, dotée de statuts réglementant les conditions d'accès au métier, la fabrication et la vente des produits, et pourvue de représentants (bayles) responsables devant l'administration communale. À Toulouse, les métiers ainsi définis n'apparaissent qu'à la fin du XIII siècle. Les bouchers ne possèdent pas de statuts avant le $\mathrm{XIV}^{\mathrm{e}}$ siècle.

Cependant, la réglementation de leur profession commence à se préciser dès 1184 : une ordonnance émanant du viguier comtal, des capitouls et des bayles de la confrérie du Bourg de Saint-Sernin leur interdit de vendre de la viande malsaine et de former des sociétés de plus de deux personnes, sauf si s'ajoute le frère, le père ou le fils d'un des associés, afin de lutter contre toute forme d'entente pouvant provoquer une augmentation des prix ${ }^{10}$. De même, le bénéfice que les bouchers peuvent tirer de leurs ventes est limité au douzième de la somme.

Cette mesure s'applique aussi à d'autres professions : un établissement promulgué en 1152 par le conseil municipal ${ }^{11}$ limite le bénéfice des boulangers à quatre deniers par carton de froment et celui des meuniers à un seizième de la mouture. Ce texte légifère par ailleurs sur la revente de plusieurs denrées (vin, blé, avoine, noix, huile, oignon, poisson, fruits) en limitant les achats des revendeurs pour éviter qu'ils n'accaparent toute la marchandise et fassent grimper les prix.

À la lecture des sources du $\mathrm{XII}^{\mathrm{e}}$ siècle, il semblerait que les seules denrées dont la fabrication et la vente dépendent de professionnels soient le pain et la viande. L'ordonnance de 1184 précédemment citée marque un début d'organisation de la profession de boucher : en s'associant entre eux, ses membres développent des solidarités pouvant conduire à l'émergence d'une conscience de groupe.

$\mathrm{Au} \mathrm{XIII}{ }^{\mathrm{e}}$ siècle, le contexte politique chaotique (guerre albigeoise suivie par l'intégration du comté à la Couronne de France en 1271) entraîne une lacune dans les sources. Il faut attendre le siècle suivant pour voir apparaître des métiers tels que nous les avons définis cidessus.

\section{L'institution de métiers spécialisés aux $X I V^{\mathrm{e}}-X V^{\mathrm{e}}$ siècles}

\footnotetext{
${ }^{8}$ Comprenant une partie de la Cité sur la rive droite, elle débordait hors des murs et finissait au sud du Château Narbonnais, cf. Ramet, 2008 (1953), p. 51 et Wolff, 1986, p. 68.

${ }^{9}$ A.M.T., AA 1/11, f ${ }^{\circ} 13-14:$ : Et quisquis homo scindat carnem et vendat et faciat ministeria que facere potuerit ».

${ }_{10}^{10}$ A.M.T., AA 1/71, fo $90 \mathrm{v}^{\circ}$. Cf. Gouron, 1958, p. 344.

${ }^{11}$ A.M.T., AA $1 / 4, \mathrm{f}^{\circ} 4 \mathrm{r}^{\circ}-5 \mathrm{v}^{\circ}$.
} 
$\mathrm{Au} \mathrm{XIV}^{\mathrm{e}}$ siècle, les capitouls réglementent la préparation et la vente de la viande en donnant des statuts ${ }^{12}$ aux professionnels en charge de ces activités. Les premiers statuts toulousains sont ceux des cordiers en $1270^{13}$. Pour les métiers de l'alimentation, les plus anciens datent de 1281 pour les épiciers et de 1290 pour les boulangers ${ }^{14}$.

Les statuts des agneliers (1301) cherchent à instaurer un monopole sur la vente d'agneaux et chevreaux, mais aussi de lièvres, lapins et volaille ${ }^{15}$. Les bouchers, qui débitent les viandes de porc, mouton et bœuf, sont à leur tour dotés de statuts qui leur assurent l'exclusivité de cette activité en $1322^{16}$. Ces textes déterminent les lieux d'activité et restreignent les conditions d'accès au métier: désormais, toute personne étrangère à la ville qui voudrait s'établir comme boucher devra obtenir l'accord des capitouls et payer dix livres de petit tournois. Cette somme correspond à celle prévue en 1424 par les statuts des chandeliersoliers, qui y ajoutent une livre de cire ${ }^{17}$.

Les métiers toulousains connaissent une fermeture ${ }^{18}$ au XV ${ }^{\mathrm{e}}$ siècle. Chez les bouchers, elle est visible dès 1394 : quatre années d'apprentissage sont désormais nécessaires pour exercer la profession de boucher. Pour s'établir à son compte, il faut passer un examen et payer deux livres tournois d'entrée (le fils d'un maître est dispensé de ce paiement), et enfin disposer d'une caution de cinquante livres tournois. Les bouchers étrangers souhaitant s'établir à Toulouse doivent recevoir l'accord des bayles et payer un marc d'argent ${ }^{19}$.

D'autres professions prévoient un examen d'entrée : c'est le cas des chandeliers-oliers à partir de 1424 et des boulangers à partir de $1463^{20}$. Chez les pâtissiers, il faut payer 5 sous tournois pour entrer en apprentissage à partir de 1492. Après avoir réussi l'examen du chef d'œuvre, les compagnons admis à la maîtrise doivent verser 4 livres tournois d'entrée et offrir un dîner aux autres maîtres. Les fils de maître n'ont à payer que 2 livres et sont dispensés d'examen ${ }^{21}$.

Les pâtissiers sont les seuls à pouvoir vendre de la viande cuite, en particulier sous forme de tourtes. Leurs statuts de $1315^{22}$ visent à en assurer la qualité : la pâte doit être réalisée à partir d'une farine de qualité supérieure et ne pas être trop fine pour ne pas se briser, tandis que la viande doit être lavée et ne pas être celle d'un animal à cornes (celles de brebis, chèvre et bouc étant considérées comme des viandes de seconde qualité, voire malsaines). En 1492, les nouveaux statuts des pâtissiers précisent qu'aucun hôtelier, tavernier ou autre ne peut cuire de la viande dans une cassolette, un four à pain ou faire des pâtés et des saucisses pour les vendre.

\footnotetext{
${ }^{12}$ Ensemble de dispositions législatives applicables aux membres d'un métier.

${ }^{13}$ Ramet, 2008 (1953), p. 200.

${ }^{14}$ A.M.T., HH 65, f $56-57$. A.M.T., II 18, acte 7 (copie de 1347).

15 A.D.H.G., 1E 1264 : «Item quod nullus revenditor vel alius quiscumque comorans in Tholosa ausus sit emere pro revendendo infra decos Tholose cirogrillos, lepores, agnos, edulos vel alia volatilia nisi esset ille emptor ministerialis dicti ministerii anheliorum quousque pulsatum fuerit ad terciam ».

${ }^{16}$ A.M.T., HH 65, f ${ }^{\circ}$ 30-35.

${ }^{17}$ A.M.T., HH 66, fo 34-46 v.

${ }^{18}$ Restriction de ses conditions d'accès.

${ }^{19}$ A.M.T., HH 66, fo 421-433.

${ }^{20}$ A.M.T., HH 66, f ${ }^{\circ} 198 \mathrm{v}^{\circ}-202$.

${ }^{21}$ A.M.T., HH 66, fo $438 \mathrm{v}^{\circ}-446 \mathrm{v}^{\circ}$.

${ }^{22}$ A.M.T., HH 65, fo 24-26.
} 
La spécialisation s'accentue au $\mathrm{XV}^{\mathrm{e}}$ siècle : la vente de viandes salées est réservée aux chandeliers-oliers en 1424. En plus des salaisons, ils proposent de la graisse et du suif, du fromage et des noix. Cette gamme de produits s'élargit en 1475 : à la suite d'un litige entre les bouchers et les chandeliers, le Parlement réserve à ces deniers la vente au détail de lard, chairs salées, jambon, chandelles, moutarde, saindoux, huile d'olive et de noix ${ }^{23}$.

En 1518, un autre litige entraîne la séparation des chevrotiers et des bouchers, jusqu'alors réunis au sein du même métier ${ }^{24}$. L'acte qui y pourvoit détaille les lieux de vente autorisés pour les chevrotiers (grand'place Arnaud-Bernard, porte Saint-Étienne, faubourg du ChâteauNarbonnais, Saint-Cyprien), et les viandes attribuées à chacun des trois métiers pour qu'ils n'empiètent pas l'un sur l'autre. Ainsi, les chevrotiers se voient réserver la vente de viande issue de chevreaux castrés, brebis, boucs, truies saines et porcs ladres ${ }^{25}$; les bouchers, celle de bœuf, vache, veau, mouton et porc ; les agneliers, celle d'agneau et de chevreau.

La spécialisation des tâches au sein de la filière carnée, mise en place dès le XIV siècle, permet à ces professionnels de devenir de véritables experts dans leur métier.

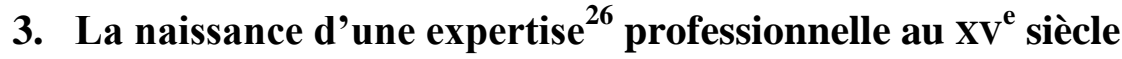

Les statuts de métiers prévoient la désignation de bayles, mentionnés dans les statuts des épiciers dès 1281. Chez les agneliers, ils sont au nombre de trois et doivent inspecter les viandes d'agneau, chevreau, lièvre, lapin et volaille vendues à Toulouse. On en trouve également chez les oliers dès $1303^{27}$ et chez les pâtissiers dès 1315 . Ce sont des experts choisis pour leur réputation d'honnêteté et leur compétence professionnelle ${ }^{28}$. Aux XIII ${ }^{\mathrm{e}}$ et XIV ${ }^{\mathrm{e}}$ siècles, ils sont nommés par les capitouls, tandis qu'au $\mathrm{XV}^{\mathrm{e}}$ siècle, ils sont recrutés soit par cooptation, soit par le suffrage des maîtres; les consuls confirment néanmoins l'élection et reçoivent leur serment de bien remplir leur charge et d'observer les statuts ${ }^{29}$. Au XV siècle, tous les métiers de l'alimentation en sont dotés. Les bayles servent d'interlocuteurs privilégiés aux autorités municipales, qui leur délèguent une partie de leur pouvoir pour contrôler les marchandises proposées à la vente et se saisir de celles dont la qualité est jugée suspecte ; les consuls décident ensuite du sort de ces produits, qu'ils peuvent détruire ou faire distribuer aux pauvres selon les cas.

Les bayles des bouchers sont mentionnés pour la première fois dans les statuts de 1394. Ils vérifient la provenance et la qualité des viandes lorsque celles-ci sont sujettes à caution, et ils inspectent les animaux avant l'abattage; ils peuvent également infliger des amendes aux bouchers fraudeurs.

\footnotetext{
${ }^{23}$ A.M.T., HH 66, fo 51-54.

${ }^{24}$ A.M.T., AA 3/317, p. 454-455 et AA 5/383 (non daté) ; A.D.H.G., 1E 1264 (1518).

25 «cabras crestatz, oveilles et manos colhars, tercias sannados et porcs meselz ».

${ }^{26}$ Bruno Laurioux propose une définition volontairement large de l'expertise en matière alimentaire : « elle désigne la capacité d'un individu, acquise par l'étude et/ou l'expérience et reconnue par une autorité publique ou une organisation professionnelle, de préparer ou fabriquer un aliment, d'en mesurer la quantité, d'en évaluer la composition et/ou la valeur marchande, d'en reconnaître les qualités organoleptiques et/ou l'état sanitaire. Cette expertise peut [...] déboucher sur l'édiction de normes, la prescription de conseils ou la formalisation de sanctions », cf. Denjean et Feller, 2013, p. 25.

${ }^{27}$ A.M.T., HH 65, f ${ }^{\circ} 49-54$.

${ }^{28}$ Gouron, 1958, p. 177-178.

${ }^{29}$ Ramet, 2008 (1953), pp. 201-202.
} 
Les bayles des pâtissiers doivent visiter les boutiques des pâtissiers, les tavernes, les hôtelleries et autres lieux où sont vendues des chairs rôties ou bouillies. Ils sont accompagnés par un sergent et ils peuvent faire ouvrir coffres, caisses et armoires pour se saisir des viandes suspectes. Après les avoir examinées, ils doivent faire un rapport aux consuls si elles se révèlent mauvaises ${ }^{30}$.

Les bayles sont parfois secondés par des «prud'hommes». D'après les statuts des bouchers de 1394, quiconque souhaite s'établir à son compte doit passer un examen devant six prud'hommes désignés par les bayles. Les bayles des ortolans (vendeurs de légumes) peuvent quant à eux se faire assister par deux prud'hommes du métier ${ }^{31}$. Ce terme semble correspondre à des membres du métier particulièrement expérimentés, mais peut concerner des officiers municipaux choisis pour superviser le travail des bayles. Ainsi, les statuts des bouchers de 1422 prévoient la désignation de quatre prud'hommes pour surveiller la vente de la viande ${ }^{32}$. En 1474, le Parlement décide de nommer deux prud'hommes pour accompagner les bayles des bouchers dans leurs visites de contrôle quotidiennes ${ }^{33}$. Il n'est pas dit s'il s'agit de membres du métier, et l'on peut se poser la question de leur niveau d'expertise.

Les bayles d'un métier peuvent réclamer des statuts aux autorités sous couvert d'assurer la qualité des produits face à une concurrence frauduleuse : en 1301, les agneliers se plaignent des vendeurs étrangers dont les pratiques nuisent à l'image de leur profession, et réclament le monopole de la vente de viande d'agneau, chevreau, lièvre, lapin et volaille afin d'assurer aux consommateurs la qualité de ces produits. De même, les chandeliers reçoivent de nouveaux statuts en 1475 après un litige avec les bouchers qui s'étaient mêlés de vendre du suif blanc.

Les capitouls imposent parfois des statuts pour mettre fin à des pratiques malhonnêtes ${ }^{34}$. En 1322, ils convoquent l'ensemble des bouchers ainsi que des prud'hommes extérieurs à la profession pour rédiger des statuts empêchant les fraudes que des habitants ont dénoncées. Par contre, en 1394, la demande vient des bayles auxquels se sont associés plusieurs membres du métier, dont les noms sont précisés en préambule. Les règles élaborées touchent à de nombreux sujets : rapports avec les écorcheurs et les abatteurs, mesures d'hygiène, conditions d'entrée dans le métier, exigences de qualité, achats et vente d'animaux, rôle des bayles. Leur précision montre une parfaite connaissance du métier de la part des rédacteurs.

Ainsi, les métiers de la viande connaissent une spécialisation poussée qui permet l'émergence d'experts, ce qui n'est pas le cas pour la vente du vin.

\section{La vente du vin : une activité méconnue et peu professionnalisée}

À Toulouse, n'importe qui peut vendre du vin : il n'existe que peu de spécialistes de cette activité, qui n'a par ailleurs guère laissé de sources.

\section{Un commerce fragmenté entre une grande variété d'acteurs}

\footnotetext{
${ }^{30}$ A.M.T., HH 66, $\mathrm{f}^{\circ} 438 \mathrm{v}^{\circ}-446 \mathrm{v}^{\circ}(1492)$.

${ }^{31}$ A.M.T., HH 66, f ${ }^{\circ} 24-26 \mathrm{v}^{\circ}$ (1464).

${ }^{32}$ A.D.H.G., 1E 1264.

${ }^{33}$ A.D.H.G., 1B $4, \mathrm{f}^{\circ} 26 \mathrm{r}^{\circ}-\mathrm{v}^{\circ}$.

${ }^{34}$ Le préambule de la plupart des statuts souligne par ailleurs la volonté des capitouls de lutter contre les mauvaises pratiques et d'œuvrer en faveur du bien public.
} 
En 1141, un privilège du comte de Toulouse Alphonse Jourdain ${ }^{35}$ donne un aperçu des acteurs impliqués dans ce commerce. Les habitants de la ville et du faubourg de Toulouse peuvent vendre leur vin en toutes saisons sans payer de taxe, contrairement aux forains (personnes extérieures) qui n'ont pas le droit de vendre leur vin au détail ; seul le commerce de gros leur est permis. Les revendeurs de la ville qui vont faire des achats à l'extérieur paient un denier par charge importée mais peuvent vendre le vin au détail.

$\mathrm{Au} \mathrm{XII}^{\mathrm{e}}$ siècle, aucun professionnel de la vente du vin n'est mentionné dans les sources, toutefois peu nombreuses. À cette époque, la plupart des activités liées à la préparation et à la vente de denrées alimentaires semblent être le fait de non-spécialistes. Elles le restent probablement jusqu'au $\mathrm{XV}^{\mathrm{e}}$ siècle, date à laquelle de nombreux métiers se dotent de statuts, tels que les ortolans et les marchands de poisson salé ${ }^{36}$.

Des établissements religieux pratiquent la vente de vin malgré l'interdiction de la papauté : le cardinal Romain de Saint-Ange, légat du pape, rappelle en 1233 la défense faite aux moines de vendre du vin dans les dépendances du couvent ${ }^{37}$. Les collèges universitaires s'adonnent aussi à ce commerce, mais refusent de payer les taxes (souquet) qui lui sont associées $^{38}$. En 1393, l'Université se lance dans un procès contre les capitouls ${ }^{39}$ et parvient à être exemptée du souquet en 1415, contrairement aux autres ecclésiastiques ${ }^{40}$.

Enfin, des bourgeois peuvent s'associer à des taverniers à qui ils prêtent de l'argent ou procurent du vin : en 1414, la veuve du sire Pierre Robert accorde 100 livres de petit tournois au tavernier Arnaud d'Arfeuil, qui partagera avec elle la moitié des bénéfices issus de cet investissement ${ }^{41}$.

Nous ne savons malheureusement pas quelle était la part de ces non-spécialistes dans la vente du vin à Toulouse, contrairement à Orvieto qui a conservé les registres de paiement des taxes portant sur le vin. Ces registres montrent que les particuliers n'intervenaient que de manière ponctuelle, l'essentiel de ce commerce étant entre les mains des taverniers qui formaient un métier ${ }^{42}$. À Toulouse il existe aussi des taverniers, mais ils ne sont pas dotés de statuts.

\section{Des spécialistes évoluant en dehors du cadre des métiers}

Les taverniers font partie des rares spécialistes de la vente du vin mentionnés par les sources. On les rencontre pour la première fois en 1340, dans une ordonnance prohibant la vente et l'importation à Toulouse de vins frelatés ${ }^{43}$; ils y sont associés à des vinaterii (marchands de vin). Philippe Wolff évalue à environ 70 le nombre des auberges et hôtelleries toulousaines vers le milieu du $\mathrm{Xv}^{\mathrm{e}}$ siècle $^{44}$. À Bordeaux, Sandrine Lavaud fait état d'une trentaine de tavernes, soit une pour 600 à 700 habitants, sachant que les taverniers n'ont pas le

\footnotetext{
${ }^{35}$ A.M.T., AA $1 / 1, \mathrm{f}^{\circ} 1-2$.

${ }^{36}$ A.M.T., HH 66, $\mathrm{f}^{\circ} 24-26 \mathrm{v}^{\circ}(1464)$ et $159 \mathrm{v}^{\circ}-161(1465)$.

${ }^{37}$ A.M.T., AA 5/7, p. 94.

${ }^{38}$ Foissac, 2010, p. 491-494.

${ }^{39}$ A.M.T., II 70, acte 28.

${ }^{40}$ A.M.T., BB 2, p. 8.

${ }^{41}$ Verdon, 2002, p. 231.

${ }^{42}$ Riccetti, 1994, p. $145-151$ et 161-162.

${ }^{43}$ A.M.T., AA 5/156, p. 722-727.

${ }^{44}$ Wolff, 1978, p. 97.
} 
monopole de la vente du vin : les bourgeois ont aussi le droit de vendre au détail le vin de leur domaine $^{45}$. À Paris, tout le monde peut être tavernier, à condition de posséder assez d'argent pour payer l'impôt des mesures - un denier par jour - et l'office des crieurs - quatre deniers la journée ${ }^{46}$.

Les crieurs sont mentionnés à Toulouse par un statut communal qui leur défend en 1221 d'indiquer la provenance géographique (lieu, ville ou château) du vin dont ils font la réclame ; ils ne doivent mentionner que son prix et par qui il est vendu ${ }^{47}$. Cette activité est mieux renseignée à Paris, où les crieurs contrôlaient la préparation du vin et en fixaient le prix en accord avec le tavernier, jouant ainsi le rôle d'experts, avant d'aller faire leur criée en proposant des échantillons aux passants ${ }^{48}$.

Ces fonctions sont méconnues car elles ne sont pas réglementées par des statuts. Il faut attendre 1525 pour trouver des réceptions de maîtrise pour les hôteliers, qui vendent - entre autres - du vin à leurs clients. Ce sont des listes établies chaque année par les capitouls pour recenser les nouveaux maîtres ayant prêté serment devant eux à leur entrée en fonction. Il n'y en a pas pour les taverniers, mais les deux métiers sont parfois confondus : ainsi, le tavernier Pierre du Pont, hôte à l'enseigne du Pilier Vert, est reçu dans le métier des hôteliers de Toulouse en $1526^{49}$ et il apparaît à nouveau comme tavernier en $1529^{50}$. Lors de la réception du tavernier Jean Flesche, hôte à l'enseigne du Sauvage Vert, dans le métier des hôteliers en 1527, le tavernier Pierre Caysson se porte caution; ce dernier est reçu à son tour dans ce même métier en 1529, à l'enseigne des Saints Innocents ${ }^{51}$.

Il faut toutefois noter que certains taverniers exerçaient un deuxième métier en parallèle, tel Jean Vayssière, hôtelier des Trois Rois et boulanger, qui teste en $1447^{52}$, ou le tavernier Stéphane de Fonte, reçu dans le métier des chandeliers de Toulouse en $1466^{53}$. Cette pluriactivité, bien connue dans les campagnes médiévales et modernes, a été encore peu étudiée dans les sociétés urbaines de la fin du Moyen Âge. Des différences pouvaient exister entre l'activité proclamée d'un artisan et son activité réelle, beaucoup plus diversifiée ${ }^{54}$.

À Toulouse, cette pluriactivité semble de moins en moins tolérée. Une ordonnance municipale interdit à partir de 1465 d'exercer deux métiers et impose aux professions non encore réglées de se doter de statuts ${ }^{55}$. Cependant, on ne trouve pas trace de statuts pour les activités liées au vin, ni avant ni après cette date. La profession de tavernier demeure donc libre, comme à Bordeaux où les taverniers sont seulement astreints à prêter serment tous les ans ${ }^{56}$.

Face à de telles différences entre le commerce du vin et celui de la viande, il paraît nécessaire de chercher des éléments d'explication.

\footnotetext{
${ }^{45}$ Lavaud, 2003, p. 87-89.

${ }^{46}$ Verdon, 2002, p. 231-233.

${ }^{47}$ A.M.T., AA $1 / 93, \mathrm{f}^{\circ} 111$.

${ }^{48}$ Verdon, 2002, p. 232-233.

${ }^{49}$ A.M.T., HH 78, $\mathrm{f}^{\circ} 40 \mathrm{r}^{\circ}$.

${ }^{50}$ A.M.T., BB 73, p. 131-133.

${ }^{51}$ A.M.T., HH 78, $\mathrm{f}^{\circ} 61 \mathrm{v}^{\circ}$ et $96 \mathrm{v}^{\circ}$.

${ }^{52}$ Wolff, 1978, p. 102.

${ }^{53}$ A.M.T., HH 76, f ${ }^{\circ} 33 \mathrm{r}^{\circ}$.

${ }^{54}$ Pfirsch, 2002, p. 5-6.

${ }_{55}$ A.M.T., AA 5/70, p. 404-407.

${ }^{56}$ Lavaud, 2003, p. 87.
} 


\section{Quelques pistes d'interprétation}

Le commerce de la viande est perçu par les autorités municipales comme une activité particulièrement sensible, devant être confiée à des spécialistes identifiés, aux compétences reconnues. La vente de vin ne semble au contraire pas nécessiter de contrôles importants. Cette différence tient à plusieurs facteurs d'explication.

\section{La dépendance envers le marché}

La non-professionnalisation de la vente du vin s'explique par le type de vin consommé à Toulouse : il s'agit d'un vin local qui n'est pas doté d'un prestige particulier. Beaucoup de Toulousains en produisent et n'en achètent pas pour leur consommation personnelle, sauf les plus pauvres qui ne possèdent pas de vignes. Ces derniers doivent se procurer du vin au détail auprès des taverniers et payer les taxes attachées à ce produit, soit le souquet instauré en 1350 pour financer les travaux de fortifications pendant la guerre de Cent Ans ${ }^{57}$.

Au contraire, malgré la présence d'un élevage urbain difficile à estimer, les habitants de la ville dépendent en grande partie du marché pour s'approvisionner en viande. En 1322, il y a 177 bouchers pour un total de 40000 habitants, soit 1 boucher pour 226 habitants. Ils sont répartis à travers toute la ville : 39 rue des Bancs Mages, 12 place Saint-Étienne (devant la cathédrale), 9 place Montaygon, 11 rue Peyrolières, 18 aux Bancs des Carmes, 7 aux Bancs de la Dalbade, 14 aux Bancs du Salin, 6 entre les portes du château Narbonnais, 20 près du palais épiscopal, 16 place Saint-Sernin, 4 au gué du Bazacle, 21 à Saint-Cyprien. En 1398, un registre de perception fiscale mentionnant tous les chefs de famille permet d'identifier 94 bouchers pour une population tombée à environ 25000 habitants en raison des pestes et des disettes, soit au moins un boucher pour 266 habitants $^{58}$.

La consommation de viande augmente au siècle suivant, conduisant les capitouls à fixer un prix maximum pour éviter que l'écart entre l'offre et la demande ne le fasse trop enchérir. En Castille, Provence et dans le royaume de Valence, un prix maximum est aussi imposé par la municipalité car la viande est vendue au poids ; au contraire, à Rouen ou Paris, elle est vendue à l'estime et les autorités ne peuvent donc pas contrôler la variation des prix. Cependant, au début du XVI ${ }^{\mathrm{e}}$ siècle, les villes du Nord adoptent la vente au poids et la fixation de prix maximum pour enrayer l'inflation ${ }^{59}$.

À Toulouse, si les prix baissent entre 1436 et 1470, ils ne cessent ensuite d'augmenter : en 1436, le bœuf vaut 6 deniers la livre, le mouton 9 ; en 1470, le bœuf vaut 5 deniers la livre, le mouton 7 ; en 1473, le bœuf vaut 10 deniers la livre, le mouton 14 ; en 1497, le bœuf vaut 12 deniers la livre, le mouton $16^{60}$. Le prix de la viande demeure très élevé au début du XVI siècle, atteignant en 152916 deniers pour le bœuf, 2 sous pour le mouton et 3 sous pour le

\footnotetext{
${ }^{57}$ A.M.T., II 61, acte 13.

${ }^{58}$ Wolff, 1978, p. 108-110. Cf. carte réalisée à partir du plan Tavernier, A.M.T., II 671.

${ }^{59}$ Banegas López, 2012, p. 162.

${ }^{60}$ A.M.T., BB 7, f ${ }^{\circ}$ 17. A.D.H.G., 1 B 3, $\mathrm{f}^{\circ} 292$ et 483. A.D.H.G., 1 B 10, $\mathrm{f}^{\circ} 23$. Il s'agit des prix maximum fixés par les autorités.
} 
veau $^{61}$. La même situation a été constatée à Tours par Bernard Chevalier, qui établit un parallèle avec la hausse de la consommation carnée ${ }^{62}$.

La réglementation des prix a été beaucoup plus précoce pour le poisson : en 1181 et 1182 , deux statuts communaux fixent un prix maximum pour le saumon, les truites, les lamproies, les anguilles, les chabots et les ablettes ${ }^{63}$. Pour le pain et l'huile, il existe une échelle mobile des prix, indexée sur celui de l'émine de blé ou de noix ${ }^{64}$.

Dans la vente du vin, il faut attendre le $\mathrm{XVI}^{\mathrm{e}}$ siècle pour voir apparaître un contrôle des prix : en 1525, une ordonnance municipale prévoit un relevé systématique des prix place Saint-Georges ${ }^{65}$, effectué par «un homme de bien sachant lire et écrire », choisi par les capitouls $^{66}$. Il s'agit d'éviter que les prix n'enflent trop, alors que Toulouse connaît une crise de subsistance à la fin des années 1520 . Les consuls finissent en 1528 par fixer le prix du vin, dans un contexte de pénurie générale des vivres ${ }^{67}$.

Le marché du vin étant plus réduit que celui de la viande, les autorités ne s'en préoccupent qu'en cas de crise, soit que l'approvisionnement soit insuffisant, soit que la qualité du vin menace la santé des consommateurs. Ces risques sont cependant bien plus élevés dans le cas de la viande.

\section{L'essor des préoccupations sanitaires}

La qualité du vin est moins surveillée que celle de la viande. Un seul texte se soucie de la dangerosité du vin et prohibe la vente et l'importation à Toulouse de vins frelatés auxquels seraient mêlés, pour en augmenter le poids, de la terre glaise, du sel, du vermillon, du bois brésil (de couleur rouge), de l'alun de roche, du calomel, des crottes de chien, de la chaux et autres matières dangereuses. Cette ordonnance a été prise par les capitouls en 1340, après délibération menée avec les assesseurs, les médecins (qui jouent ici le rôle d'experts) et autres prud'hommes $^{68}$.

La viande peut créer plus de risques pour la santé que le vin. La présence d'animaux malades dans la ville fait craindre la propagation d'épidémies. On redoute notamment la lèpre porcine, mais on se méfie surtout de ses effets digestifs, et non de la maladie elle-même ${ }^{69}$.

Pour contrer ces dangers, les statuts des bouchers interdisent d'abattre des animaux suspects : la viande doit provenir d'animaux tués sur place, dont l'état sanitaire est vérifié avant et après l'abattage ${ }^{70}$. Le bétail ne peut être tué en dehors des abattoirs. Des spécialistes appelés langueyeurs s'attachent à détecter les pustules sublinguales révélatrices de la ladrerie des porcs. De même, les statuts des agneliers de 1301 défendent d'abattre et de découper des agneaux ou des volatiles « fous, clavelés ou galeux » dans l'enceinte de la ville.

\footnotetext{
${ }^{61}$ A.M.T., BB 68, p. 155-156.

${ }^{62}$ Chevalier, 1993, p. 65.

${ }^{63}$ AMT, AA 1, actes 6 et 7.

${ }^{64}$ AMT, HH 65, $\mathrm{f}^{\circ} 49 \mathrm{v}^{\circ}-54$ (1303). AMT, HH 66, f $\mathrm{f}^{\circ} 34-46 \mathrm{v}^{\circ}(1424)$ et 47-47 $\mathrm{v}^{\circ}$ puis $472-477 \mathrm{v}^{\circ}$ (non daté). A.M.T., II 18, acte 7 (1290).

${ }^{65}$ A.M.T., HH 22 (1525-1557).

${ }^{66}$ A.M.T., BB $149, \mathrm{f}^{\circ} 12 \mathrm{v}^{\circ}-14 \mathrm{r}^{\circ}$.

${ }^{67}$ A.M.T., BB 150/57, p. 131-132.

${ }^{68}$ A.M.T., AA 5/156, p. 722-727.

${ }^{69}$ Ferrières, 2002, p. 29-52.

${ }^{70}$ A.M.T., HH 66, f ${ }^{\circ}$ 421-433.
} 
Écorcheurs et abatteurs n'ont pas le droit d'entretenir des porcs près des abattoirs ni dans les rues, de peur qu'ils ne mangent de la viande saignante ou pourrie. Les peaux, les tripes, le sang ne doivent pas être conservés près des abattoirs ni dans les rues ; ils doivent être enlevés le mardi et le samedi ${ }^{71}$. Il s'agit de préserver autant la propreté des rues que la salubrité des aliments pour éviter les infections.

Ces interdictions ne semblent pas toujours respectées, comme le montre leur répétition. En 1473, un arrêt du Parlement de Toulouse défend aux bouchers de tuer, tailler ou vendre des « chairs morveuses » ou «infectes»; en 1474, un nouvel arrêt vise les «chairs suspectes, vicieuses ou morveuses ». Cette interdiction est réitérée en 1479, et un deuxième arrêt défend à tous les bouchers de faire entrer dans la ville du bétail mort, malade ou morveux. Le Parlement ordonne aux bayles des bouchers de ne tolérer aucune fraude lors de leurs contrôles et de faire observer correctement les statuts, sous peine d'une lourde amende et de privation de leur office ${ }^{72}$. Cette répétition marque aussi la volonté du Parlement d'intervenir davantage dans la police des métiers. Dans la deuxième moitié $d u X^{\mathrm{e}}$ siècle, il se livre à une sorte de combat contre les capitouls qu'il accuse de laxisme, afin d'affirmer son pouvoir face au leur ${ }^{73}$.

Les bouchers n'ont pas non plus le droit de vendre de la viande cuite ou salée de peur qu'ils n'en profitent pour écouler les chairs invendables. Ce commerce est réservé aux pâtissiers et aux chandeliers, dont les statuts visent à empêcher ce type de pratique. Les chandeliers ne peuvent vendre des salaisons de qualité insuffisante, lépreuses, putréfiées ou corrompues sous peine de confiscation et d'une forte amende. Les pâtissiers n'ont pas le droit de faire des saucisses à partir de la chair de porcs femelles, de chairs lépreuses ou d'autres chairs mauvaises et puantes. Il est également interdit de refaire cuire la viande et les pâtés ${ }^{74}$.

Les contrôles portant sur la viande ne visent pas seulement à éviter les risques sanitaires : la consommation carnée est un moyen d'affirmer son statut social et la qualité des viandes doit être certifiée, ce qui ne semble pas être le cas du vin local.

\section{Un facteur de distinction sociale}

Au Moyen Âge, la viande est perçue comme un marqueur social : selon le milieu auquel on appartient, on ne consomme pas celle des mêmes bêtes. Les statuts des bouchers de 1394 font la distinction entre les viandes nobles (bœuf, porc, mouton, veau) vendues au mazel ou sur des tables hautes devant l'église Saint-Georges, et «les mauvaises viandes salées, lépreuses ou celles des truies » et celles des brebis (la viande mâle étant réputée meilleure que la viande femelle). Celles-ci ne doivent pas être vendues sur la place Montaygon, mais sur des tables posées par terre et devant l'hôpital Sainte-Marie ${ }^{75}$. Au XVI ${ }^{\mathrm{e}}$ siècle, ce sont les chevrotiers qui se chargent de ce commerce.

Par ailleurs, la viande avariée confisquée par les bayles est distribuée aux pauvres lorsque les consuls ne la considèrent pas trop dangereuse ; dans le cas contraire, ils peuvent décider de la brûler ou de la jeter dans la Garonne. La vente des viandes des bêtes malades, blessées,

\footnotetext{
${ }^{71}$ Ibid.

${ }_{72}^{72}$ A.D.H.G., 1B $3 \mathrm{f}^{\circ} 485 \mathrm{v}^{\circ} ; 1 \mathrm{~B} 4, \mathrm{f}^{\circ} 26 \mathrm{r}^{\circ}-\mathrm{v}^{\circ} ; 1 \mathrm{~B} 5$, p. $142 ; 1 \mathrm{~B} 9$.

${ }^{73}$ Dognon, 1895, p. 479.

${ }^{74}$ A.M.T., HH 66, fo $34-46 \mathrm{v}^{\circ}$ (1424) et $\mathrm{f}^{\circ} 438 \mathrm{v}^{\circ}-446 \mathrm{v}^{\circ}$ (1492).

${ }^{75}$ A.M.T., HH 66, fo $421-433$.
} 
n'est pas formellement prohibée : elle peut se faire dans certains endroits, avec l'obligation de prévenir l'acheteur. Selon la diététique de l'époque, les pauvres possèdent un estomac capable de digérer ce type de denrée, tandis que les gens d'étude et de loisirs doivent absorber des nourritures subtiles et légères favorisant le travail de digestion ${ }^{76}$.

Ce système à trois vitesses se retrouve à Barcelone : dans le premier tiers du XIV ${ }^{\mathrm{e}}$ siècle, il $\mathrm{y}$ a dans la ville trois mazels où l'on tue et découpe mouton, cabri, bœuf, vaches, veaux et certains porcs. Il y a aussi deux mazels de viandes secondaires où se vendent boucs, chèvres, brebis, truies et porcs venant de Minorque et Toulouse. Les viandes malsaines (issues de bêtes mortes avant leur entrée dans la cité, ou malades au moment d'être abattues) et les abats des viandes secondaires sont vendues sur deux étaux situés aux portes de la ville, de l'autre côté de la muraille ${ }^{77}$.

Dans le cas du vin, il est plus difficile de distinguer la consommation des riches de celle des classes populaires. Les pièces à l'appui des comptes des repas offerts par les capitouls mentionnent divers types de vin: vin blanc, clairet ou rouge, hypocras (vin épicé sucré), muscat et muscadel. Elles ne précisent cependant pas leur provenance, et nous ne savons pas si l'on recourait à l'importation de vins plus réputés pour certaines occasions, ou si l'on se fournissait indifféremment dans l'aire habituelle d'approvisionnement - qui s'étendait jusqu'à une cinquantaine de kilomètres autour de la ville, principalement en Lauragais et Albigeois jusqu'à Gaillac ${ }^{78}$. Les étudiants du collège de Périgord buvaient surtout du vin rouge provenant des vignobles du collège, plus rarement du vin blanc ou clairet ${ }^{79}$.

À Carpentras, la consommation de vin est plus différenciée: l'évêque boit des vins liquoreux de Malvoisie et de muscat ainsi que des vins épicés, tandis que les riches marchands préfèrent des clairets et des rouges des petites villes environnantes. Les artisans consomment les vins blancs locaux et les ouvriers, de la piquette ${ }^{80}$. Les sources toulousaines ne nous donnent malheureusement pas de telles précisions.

Le commerce du vin et celui de la viande diffèrent profondément à Toulouse. La filière carnée s'est professionnalisée assez tôt, les autorités cherchant à contrôler ce secteur sensible tant à cause des risques qu'il pouvait présenter pour la santé des consommateurs qu'en raison de la place croissante occupée par la viande dans l'alimentation des habitants. Ceux-ci dépendant essentiellement du marché pour s'approvisionner, la vente de la viande était très surveillée et a fait l'objet d'une production pléthorique de textes réglementaires visant à l'encadrer, tandis qu'une expertise se constituait peu à peu au sein du métier et en dehors. Ce n'est pas le cas de la vente du vin, qui est restée très libre jusqu'au $\mathrm{XVI}^{\mathrm{e}}$ siècle et demeure encore largement méconnue à cause du manque de sources pour l'étudier.

Le commerce de la viande présente une forme originale à Toulouse de par sa division entre plusieurs métiers (bouchers, agneliers, chevrotiers, mais aussi pâtissiers et chandeliers), qui ne semble pas aussi poussée dans des villes comme Paris et Barcelone. La corporation des

\footnotetext{
${ }^{76}$ Ferrières, 2002, p. 72-73 et 82-84.

${ }^{77}$ Banegas López, 2007, p. 191 et 203.

${ }^{78}$ Higounet, 1971, p. 106.

${ }^{79}$ Alamy, 1969, p. 42-45.

${ }^{80}$ Ibid., p. 134.
} 
bouchers toulousains jouit d'une certaine autonomie dans l'élaboration de ses statuts, mais ses membres sont tout de même supervisés par des officiers municipaux. Toulouse présente un cas intermédiaire entre les villes du Nord de la France, où les corporations de bouchers se chargent elles-mêmes des contrôles, et celles de la péninsule ibérique, où ce rôle est tenu par les autorités municipales ${ }^{81}$.

Clémentine Stunault

Université Toulouse II - Jean Jaurès

${ }^{81}$ Banegas López, 2012, p. 175 et 185. 